\title{
MASTER-SAAO transient detections: new Cataclysmic Variable discoveries
}

\section{A. H. Buckley ${ }^{1}$}

South African Astronomical Observatory, PO Box 9, Observatory 7935, Cape Town, South Africa E-mail: dibnobasaao.ac.za

\section{H. Breytenbach}

South African Astronomical Observatory, PO Box 9, Observatory 7935, Cape Town, South Africa \& Department of Astronomy, University of Cape Town, Private Bag X3, Rondebosch 7701, South Africa E-mail: hannesasaao.ac.za

\section{A. Kniazev}

South African Astronomical Observatory, PO Box 9, Observatory 7935, Cape Town, South Africa E-mail: akniazevesaao.ac.za

\section{M. Kotze}

South African Astronomical Observatory, PO Box 9, Observatory 7935, Cape Town, South Africa E-mail: marissalsaao.ac.za

\section{Motsoaledi}

South African Astronomical Observatory, PO Box 9, Observatory 7935, Cape Town, South Africa \& Department of Astronomy, University of Cape Town, Private Bag X3, Rondebosch 7701, South Africa E-mail: eni hkomegmai I. com

\section{S. B. Potter}

South African Astronomical Observatory, PO Box 9, Observatory 7935, Cape Town, South Africa E-mail: sbpesaao.ac.za

\section{P. A. Woudt}

Department of Astronomy, University of Cape Town, Private Bag X3, Rondebosch 7701, South Africa E-mail: pwoudteast.uct.ac.za

\section{Lipunov}

Sternberg Astronomical Institute,

Lomonosov Moscow State University, Universitetskiy pr. 13, Moscow 119992,

Russia

E-mail: Iipunov2007@gmail.com

\section{E. Gorbovskoy}

Sternberg Astronomical Institute,

Lomonosov Moscow State University, Universitetskiy pr. 13, Moscow 119992,

Russia

E-mail: gorbovskoylsai.msu.ru

${ }^{1}$ Speaker 
The detection of optical transients is escalating, particularly with the advent of deeper, faster and wider panchromatic imaging surveys of the sky. Future optical transient detection systems, like those of PanSTARRs and LSST, will result in an explosion of transient alerts, probably up to $\sim 10^{6}$ alerts per night. Over the last $\sim 7$ years or so, several wide field optical imaging surveys have begun from which new transient detections are continually being made. They include CRTS, MASTER, PTF and ASAS-SN, the latter two aimed at discovering new Supernovae. These have begun to be supplemented by larger scale surveys, like PanSTARRs, GAIA and SkyMAPPER, which although not dedicated to detecting transients, will nonetheless discover many. In this paper we report on the first local optical transient detection system established at the SAAO Sutherland station, namely MASTER-SAAO, the first southern hemisphere node of the Russian-based MASTER II network. The characteristics of the MASTER-SAAO system are described and the parameters of the survey compared to the CRTS, which is the only other transient survey for which comprehensive results on Cataclysmic Variables have been published. To date MASTER-SAAO has discovered 141 non-Solar System optical transients (plus 2 comets and several likely minor planet detections). A total of $78 \%$ (111) of these are new Cataclysmic Variables, with all except two being likely Dwarf Novae (DNe). About $50 \%$ of the DNe have outburst amplitudes in excess of $4^{\mathrm{m}}$, and $20 \% \geq 5^{\mathrm{m}}$. We expect that the latter are mostly SU UMa systems, as well as some extreme amplitude WZ Sge systems (some with amplitudes of $7^{\mathrm{m}}$ ). MASTER-SAAO has a detection limit of $B \sim 19-20$, comparable to the $V_{\text {CSS }} \sim 20$ limit of the CRTS (depending on CV colour). Based on the CV detection statistics in the latter survey, we believe that MASTER-SAAO is detecting essentially the same CV population as CRTS, for a detection amplitude threshold $>2.2^{\mathrm{m}}$. We present two results of the initial follow-up program on CVs discovered by MASTER; a Dwarf Nova and a new eclipsing Polar. Finally, we discuss the current and future prospects for rapid follow-up observations at SAAO of transients and other targets of opportunity, including CVs, with new robotic telescopes like MONET-South and the new SAAO 1-m robotic telescope, both expected to be operational in 2016, as well as with SALT.

The Golden Age of Cataclysmic Variables and Related Objects - III, Golden2015

7-12 September 2015

Palermo, Italy 


\section{Introduction}

The study of astrophysical transients has entered a new era with the establishment of a number of new ground-based facilities used in both detection and follow-up programs (e.g. for alerts from X-ray or $\gamma$-ray satellites). Some of these have targeted specific classes of transients, for example supernovae, e.g. PTF [1] and ASAS-SN [2] and Gamma Ray Bursts, e.g. ROTSE [3] and Watcher [4]. Other optical transient detection systems currently operating include the Catalina Real Time Survey (CRTS) [5] and MASTER [6], both of which have been successful in detecting a variety of transients, including Cataclysmic Variables, flare stars, long period variable stars, out-bursting blazars as well as Supernovae and GRBs. In addition, both surveys are also discovering Solar System transients, namely comets and minor planets. With the development of recent facilities (e.g. PanSTARRs [7], SkyMapper [8] and GAIA[9]) and those in the future (e.g. LSST [10]), the opportunity to study the transient Universe will be unprecedented. Indeed the volume of alerts will mean the traditional manner of conducting follow-up programs will become unmanageable and machine learning techniques will need to be employed in order to find the astrophysically interesting objects.

There is a continuing and growing interest amongst South African astronomers to study astrophysical transients, particularly those related to high energy events, like X-ray transients, GRBs, blazar and CV outbursts. Until now, these transient alerts came from ground-based facilities on other continents or from satellite missions.

In this paper we report on the establishment of the first comprehensive optical transient detection and follow-up system at the Sutherland observing site of the South African Astronomical Observatory, namely MASTER-SAAO. We review the specifications of this system and the results to date after $\sim 9$ months of operations, with emphasis on the new discoveries of $\mathrm{CVs}$, which represent close to $80 \%$ of the optical transient discoveries. Most of these are new examples of Dwarf Novae (DNe), with outburst amplitudes of up to $\sim 7^{\mathrm{m}}$. A comparison is made with the recent survey of CVs from the CRTS [11], the most comprehensive survey to date from an optical transient detection survey. Finally we also discuss the plans to exploit alerts from MASTER-SAAO and other facilities by conducting rapid follow-up observations with telescopes at the SAAO, including the Southern African Large Telescope (SALT).

\section{The MASTER Network}

The MASTER project (Mobile Astronomical System of TElescope Robots), aimed at the detection of optical transients, was established in Russia in 2002, when the first Russian robotic telescope was installed near Moscow [6]. The network has since expanded and now includes four other nodes in Russia, extending $\sim 80^{\circ}$ in longitude, and nodes in Argentina, the Canary Islands and South Africa. In late December 2014 the South African node, MASTER-SAAO, was installed at the SAAO Sutherland station.

The facilities have evolved and most now utilize the MASTER II systems, consisting of dual $40 \mathrm{~cm}$ telescopes on a common mount. The Argentina site currently only has a very wide 
field camera, while the original Moscow node is no longer operational. In Table 1 the locations of the MASTER nodes are shown, while Figure 1 shows a map with the MASTER nodes.

Table 1: Location of the active MASTER nodes

\begin{tabular}{|l|l|l|l|l|l|}
\hline Name & Country & Location & Longitude & Latitude & Altitude \\
\hline MASTER-ICATE* & Argentina & San Juan & $69^{\circ} .00 \mathrm{~W}$ & $31^{\circ} .80 \mathrm{~S}$ & $2430 \mathrm{~m}$ \\
\hline MASTER-IAC & Spain & Tenerife & $16^{\circ} .51 \mathrm{~W}$ & $28^{\circ} .30 \mathrm{~N}$ & $2390 \mathrm{~m}$ \\
\hline MASTER-SAAO & South Africa & Sutherland & $20^{\circ} .81 \mathrm{E}$ & $32^{\circ} .38 \mathrm{~S}$ & $1760 \mathrm{~m}$ \\
\hline MASTER-Kislovodsk & Russia & Caucasus & $43^{\circ} .75 \mathrm{E}$ & $42^{\circ} .52 \mathrm{~N}$ & $2067 \mathrm{~m}$ \\
\hline MASTER-Ural & Russia & Ekaterinburg & $57^{\circ} .54 \mathrm{E}$ & $57^{\circ} .03 \mathrm{~N}$ & $260 \mathrm{~m}$ \\
\hline MASTER-Tunka & Russia & Irkutsk & $103^{\circ} .07 \mathrm{E}$ & $51^{\circ} .81 \mathrm{~N}$ & $680 \mathrm{~m}$ \\
\hline MASTER-Amur & Russia & Blagoveschensk & $127^{\circ} .48 \mathrm{E}$ & $50^{\circ} .32 \mathrm{~N}$ & $260 \mathrm{~m}$ \\
\hline
\end{tabular}

* currently only consisting of a Very Wide Field camera

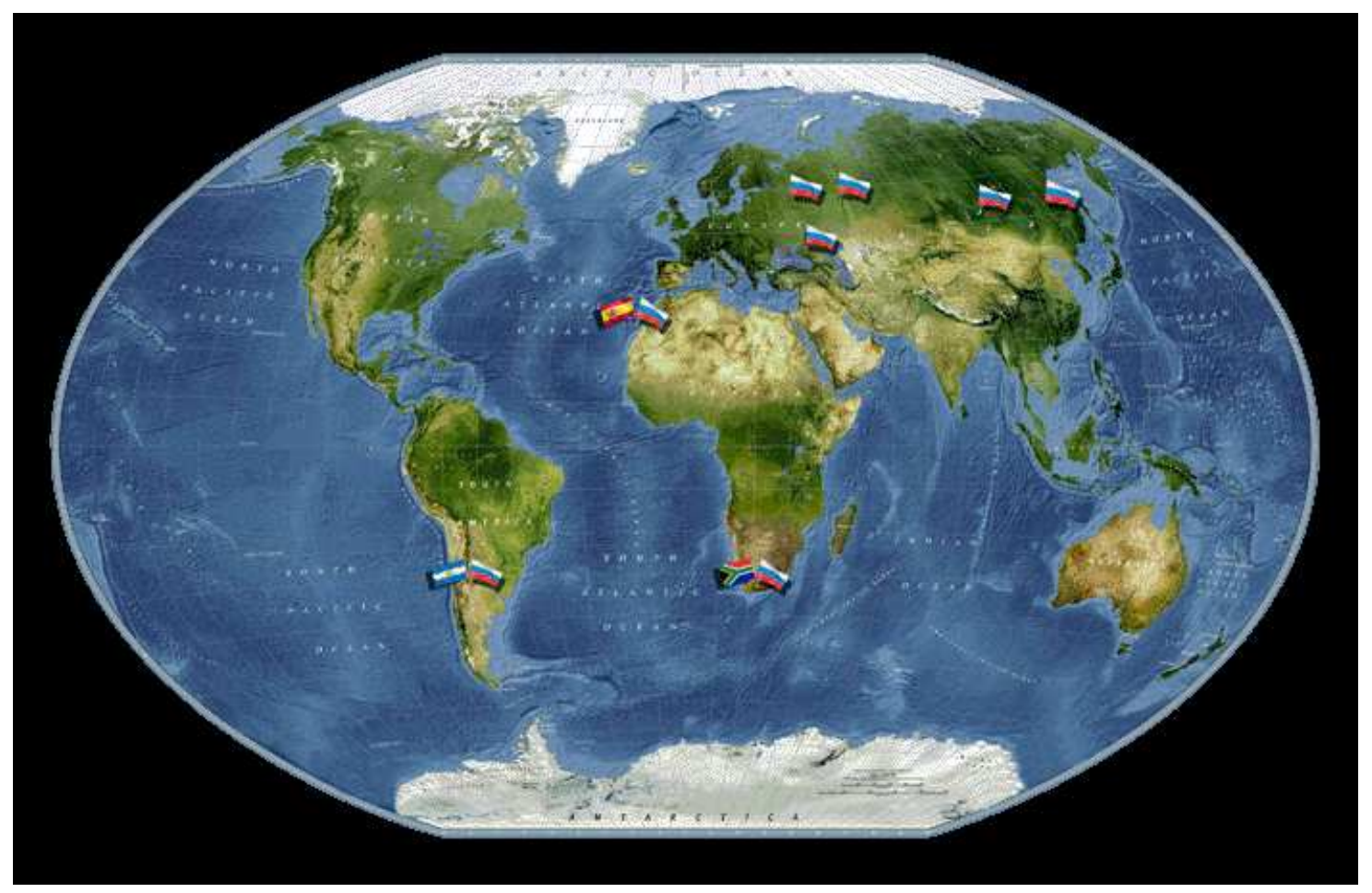

Figure 1: location of the MASTER network nodes

The main goal of the MASTER-Net project is to produce a fast and wide sky survey for optical transients down to a limiting magnitude of $19-20$. The survey is detecting a range of transients, including Galactic sources (CVs, flare stars, eclipsing binaries), AGN (blazars), supernovae and Solar System objects. All MASTER telescopes can also be guided by alerts, and have been used for the detection of prompt optical emission from GRBs, simultaneously in two filters or polarization planes. With the establishment of MASTER-SAAO, the southern 
hemisphere sky is now being routinely monitored as part of the MASTER program, for the first time.

Six of the MASTER nodes consist of identical dual $40 \mathrm{~cm}$ diameter telescopes on a common mount, with Apogee CCD cameras. The telescope tubes can either be co-aligned, each surveying an identical $2^{\circ} \times 2^{\circ}$ field, with two different filters or polarizers, or they can be misaligned to allow twice the sky coverage using identical filters. The latter is the default mode for detecting optical transients, which is done without using a filter.

\section{The MASTER-SAAO facility}

The relevant attributes of the MASTER-SAAO system and the transient survey are presented in Table 2 [6]. The telescope design for all the MASTER II systems is based on the catadioptric Hamilton design [12], utilizing a double convex entrance lens and Mangin primary mirror, followed by a small field lens. This gives a very wide field, but also a flat focal plane. A schematic of the design is shown in Figure 2, while a photograph of the MASTER-SAAO facility is shown in Figure 3.

Table 2: Characteristics of the MASTER-SAAO system

\begin{tabular}{|c|c|c|}
\hline Sub-system & Parameter & Value \\
\hline \multirow[t]{6}{*}{ Telescope } & Design & Hamilton catadioptric \\
\hline & Diameter & $40 \mathrm{~cm}$ \\
\hline & f/ratio & 2.5 \\
\hline & Platescale & $206 \mathrm{arcsec} / \mathrm{mm} ; 1.8 \mathrm{arcsec} / \mathrm{pixel}$ \\
\hline & Effective area & $940 \mathrm{~cm}^{2} /$ telescope \\
\hline & Field / telescope & $2^{\circ} \times 2^{\circ}$ \\
\hline \multirow[t]{3}{*}{ CCD camera } & Type & Apogee Alta U16M \\
\hline & Size & $4096 \times 4096$ \\
\hline & Pixel size & $9 \mu \mathrm{m}$ \\
\hline \multirow[t]{9}{*}{ Survey } & Exposure per field & $3 \times 60 \mathrm{~s}$ (grey/bright Moon) \\
\hline & & $3 \times 180 \mathrm{~s}($ dark Moon $)$ \\
\hline & Survey limiting magnitude & 19.8 dark Moon); 18.8 grey Moon \\
\hline & follow-up limiting magnitde & 22.3 (20 co-added $180 \mathrm{~s}$ exp) \\
\hline & Field of view per exposure & $2^{\circ} \times 4^{\circ}$ \\
\hline & Declination range (survey) & $-90^{\circ}$ to $\sim+25^{\circ}$ \\
\hline & Declination limit (follow-up) & $+40^{\circ}$ \\
\hline & Sky area per night* & $500-1000$ degrees $^{2}$ \\
\hline & Cadence* & $10-20$ days \\
\hline
\end{tabular}

* dependent on season, lunation 


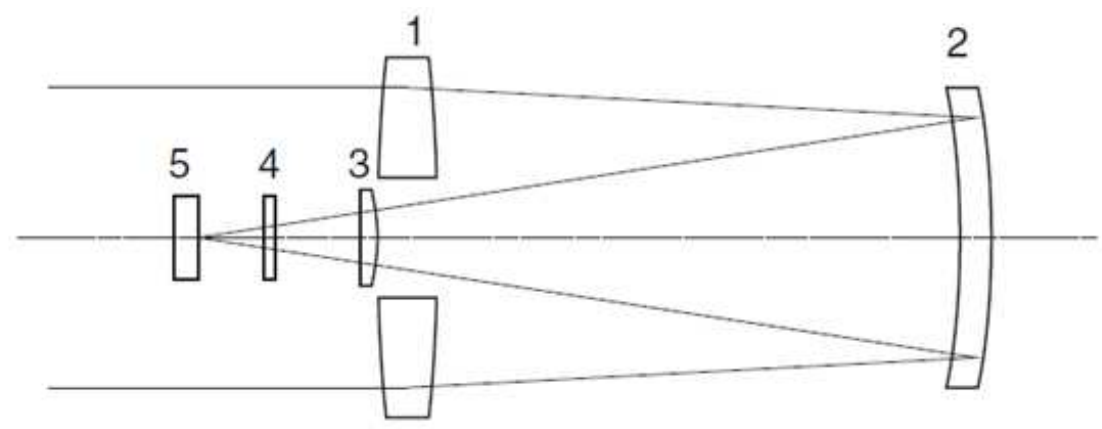

Figure 2: The Hamilton catadioptric optical design used in the MASTER II telescopes. $1=$ positive entrance lens, 2 = Mangin mirror, 3 = field lens, 4 = filter, 5 = CCD detector

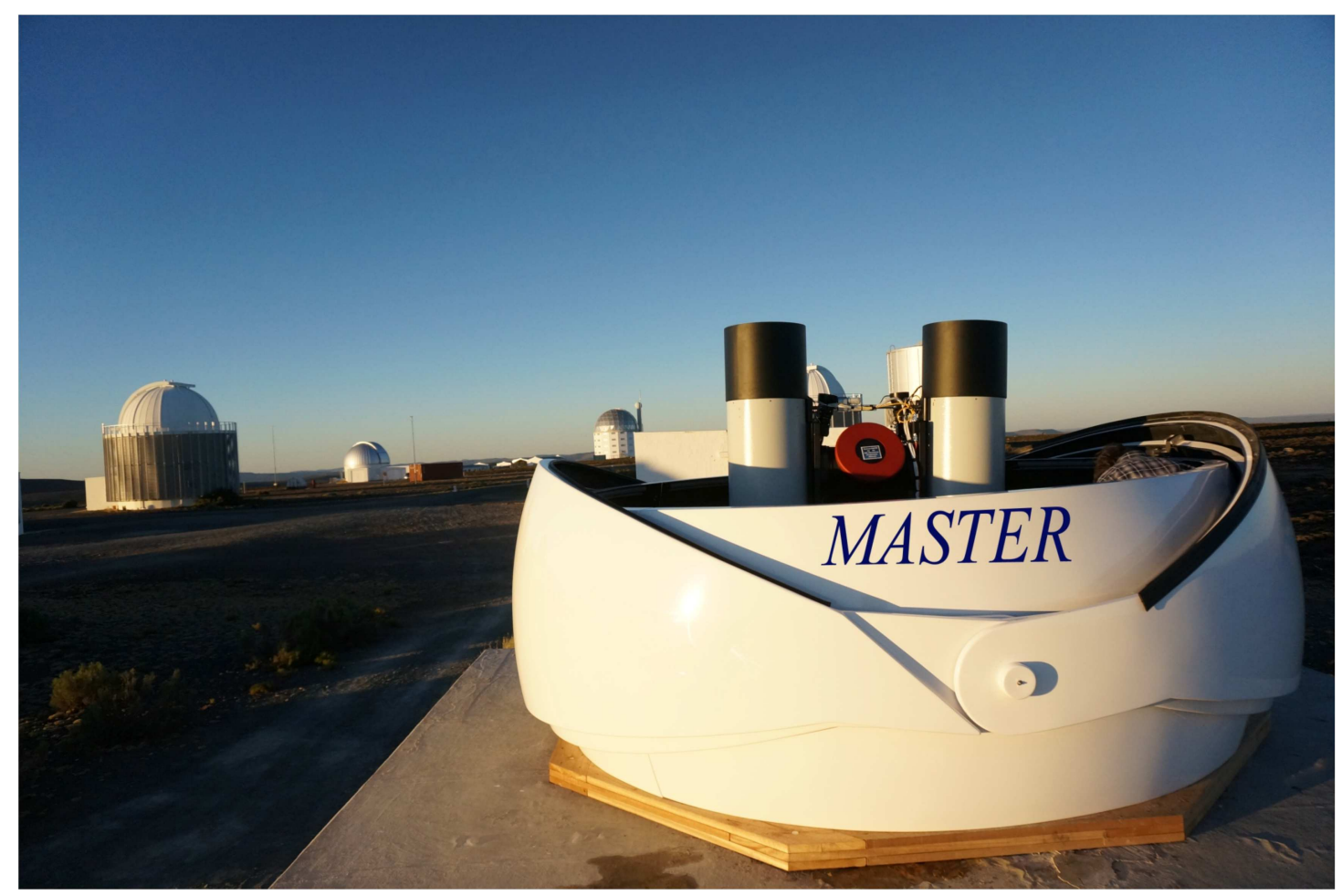

Figure 3: The MASTER-SAAO facility at Sutherland. SALT is seen in the far distance at centre.

Each telescope can observe with one of four filters $(B, V, R$ or $I)$ or with a polarizing filters, allowing measurement of Stokes $Q$ or $U$, depending on the angle of the polarizers. As well as the two $40 \mathrm{~cm}$ telescopes, each mount also has a Very Wide Field (VWF) camera, covering $\sim 1000$ degrees $^{2}$, but with a shallow limit of $V \sim 12$ in a $1 \mathrm{~s}$ exposure.

The MASTER-SAAO node was installed and commissioned at the Sutherland observing station of the SAAO over a $\sim 3$ week period, in December 2014. A clam shell dome (AstroHaven) allows rapid slewing over the entire sky, with declination range of $-90^{\circ}$ to $+40^{\circ}$, 
although the survey only extends to between $+20^{\circ}$ and $+30^{\circ}$. There is no limit on the Galactic latitude, unlike the similar CRTS survey [5], which excludes the regions within $10^{\circ}-15^{\circ}$ of the Galactic plane. However, the Galactic plane is a lower priority region, so the amount of coverage is considerably less (by a factor $\sim 10 \times$ to $20 \times$ ) than for higher Galactic latitudes.

In Table 3 we compare some of the MASTER survey parameters with those of the CRTS [5,11], which until recently has been the only other transient detection system for which a study of CVs has been made in some detail [11].

Table 3: Comparison of the attributes of the MASTER and CRTS Systems

\begin{tabular}{|l|l|l|l|l|}
\hline Parameter & $\begin{array}{l}\text { MASTER II (all } \\
\text { nodes) }\end{array}$ & $\begin{array}{l}\text { CRTS } \\
\text { (all nodes) }\end{array}$ & $\begin{array}{l}\text { MASTER- } \\
\text { SAAO node }\end{array}$ & SSS node \\
\hline Collecting area $\left(\mathrm{m}^{2}\right)$ & 3.0 & 4.7 & 0.50 & 0.39 \\
\hline Field of View $\left(\mathrm{deg}^{2}\right)$ & 48 & 13.3 & 8 & 4.2 \\
\hline Maximum area/night $\left(\mathrm{deg}^{2}\right)$ & $\sim 4000:$ & $\sim 2500:$ & $\sim 500-1000^{*}$ & \\
\hline Cadence (days) & $10-15$ & $7-10$ & $10-20$ & $7-10$ \\
\hline Exposures & $3 \times(60 \mathrm{~s}-180 \mathrm{~s}) *$ & $4 \times 30 \mathrm{~s}$ & $3 \times(60 \mathrm{~s}-180 \mathrm{~s})^{*}$ & $4 \times 30 \mathrm{~s}$ \\
\hline Typical limiting magnitude & $18-20$ & $19-20 ; 22 \S$ & $18.8-19.8$ & $19-20$ \\
\hline Threshold for alert (mags) & 2.2 & 0.65 & 2.2 & 0.65 \\
\hline
\end{tabular}

* depending on Moon phase $§$ limit for the $1.5 \mathrm{~m}$ Catalina Sky Survey in USA

Table 3 attempts to make a comparison between the entire MASTER and CRTS networks, as well as with the two systems specific to the southern hemisphere, namely the MASTER-SAAO node in South Africa and the Siding Spring System (SSS) of CRTS in Australia (now no longer operational). While we have attempted to present the best estimates of the survey parameters, there are some inevitable uncertainties or ranges due to lack of published information and the fact that some of the parameters are dependent upon season, Moon phase and observing conditions.

The two surveys use somewhat different criteria for a transient alert. In the case of CRTS, a transient is considered to be real if it is detected to be 3 times brighter than the observed scatter and at least $>0.65^{\mathrm{m}}$ brighter than in the reference image on 3 out of the 4 consecutive images. For MASTER, the criteria is that the transient has to be $>2.2^{\mathrm{m}}$ brighter than in the reference image and detected on atleast 3 consecutive images taken at the same epoch (i.e. in all images taken at a given time during the survey mode).

\section{MASTER-SAAO Results to Date}

All MASTER optical transient discoveries are listed on the MASTER website (http://observe.pereplt.ru/MASTER OT.html) and are immediately published as Astronomer's Telegrams (ATels). Any results pertaining to GRB detections are also published in GCN circulars. The MASTER website includes discovery and reference images, coordinates, magnitudes and outburst amplitudes, where there is a pre-existing pre-alert image. MASTER is one of the most successful transient detection systems, as evidenced by the fact that up to 2014 
it has been responsible for $\sim 25 \%$ of all ATel transient alerts, second only to Swift and the most of any ground-based optical alerts (CRTS is next, with $~ 7 \%$ ). A total of 885 transient discoveries have been made by the MASTER II network over the last $~ 5.7$ years, to Sep 2015 .

Since MASTER-SAAO has been operational, many southern hemisphere optical transients have been detected, while follow-up optical observations have also been undertaken by MASTER-SAAO of transient alerts from other sources, particularly GRBs and blazar outbursts. Figure 5 shows the sky coverage in the $\sim 9$ months of operations, up to early Sep 2015. In Table 4 we list the breakdown of the 141 non-Solar System MASTER-SAAO optical transients discovered so far, in terms of object class. In addition two comets and several minor planets have also been discovered.

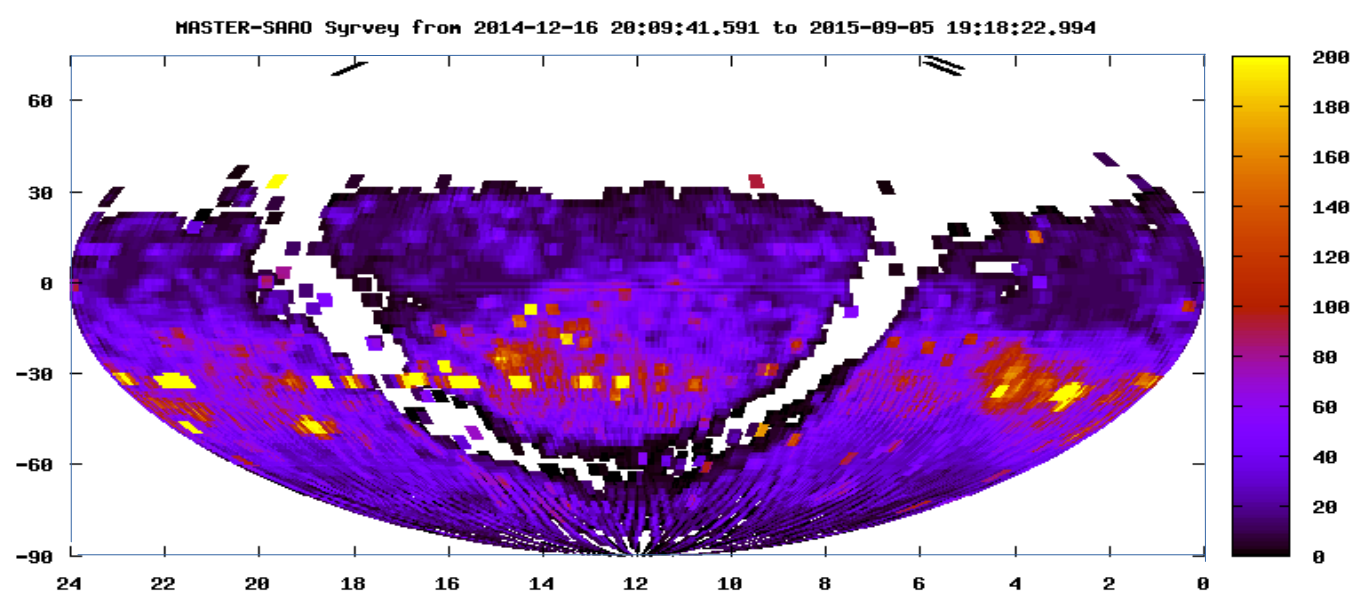

Figure 5: Sky coverage, in equatorial coordinates, of MASTER-SAAO in the $\sim 9$ months since it has been operating. The scale indicates the number of 4 degree $^{2} \mathrm{CCD}$ frames taken to date.

Table 4: Optical Transients Discovered by MASTER-SAAO (16 Dec 2014 - 5 Sep 2015)

\begin{tabular}{|c|c|c|c|}
\hline Type & Class & Number & Comments \\
\hline \multirow[t]{6}{*}{ Galactic objects } & Dwarf Novae CV in outburst & 109 & Criteria is $\Delta \mathrm{m}>2.2^{\mathrm{m}}$ \\
\hline & Novalike CV & 1 & \\
\hline & Magnetic CV (polar) & 1 & Eclipsing. Orb $\mathrm{P}=2.2 \mathrm{~h}$ \\
\hline & Flare Stars & 3 & \\
\hline & Long Period variables & 4 & \\
\hline & Anti-transient & 1 & Long period eclipser \\
\hline \multirow[t]{2}{*}{ Extragalactic } & Supernovae & 20 & \\
\hline & Gamma Ray Bursters & 2 & Detection from Swift alerts \\
\hline
\end{tabular}

In addition to the transient discoveries following from the MASTER-SAAO survey, a number of dedicated follow-up observations were also conducted, including of several GRB 
alerts (only 2 resulted in optical counterpart identifications) and blazar outburst alerts (7 observed).

Over the $\sim 9$ months that MASTER-SAAO has been operating, 141 non-Solar System optical transient discoveries have been made, of which two new CVs have been observed with SALT, plus one blazar, detected to be flaring by the MASTER-Kislovodsk node and confirmed by Fermi and Swift. Although it was planned to undertake classification spectroscopy with SALT for some of the more compelling transient detections, a proposal to do this in semester 2015-1 (from 1 May 2015) was unsuccessful, so the only observations done to date have been through an allocation of $\sim 6700 \mathrm{~s}$ of SALT Directors Discretionary Time (DDT). From 1 Nov 2015, an approved program of follow-up SALT spectroscopy began, which will run for two semesters (until 31 Oct 2016). Below we summarize the initial results of the three DDT observations, all of which are in various stages of analysis and write up as refereed journal papers.

In Figure 6 we show the distribution of all 886 MASTER transients discovered over the 5.75 years since the MASTER II networks began operating, in 2010, inclusive of all the transients discovered by MASTER-SAAO in the $\sim 9$ months it has been operational (since late Dec 2014).

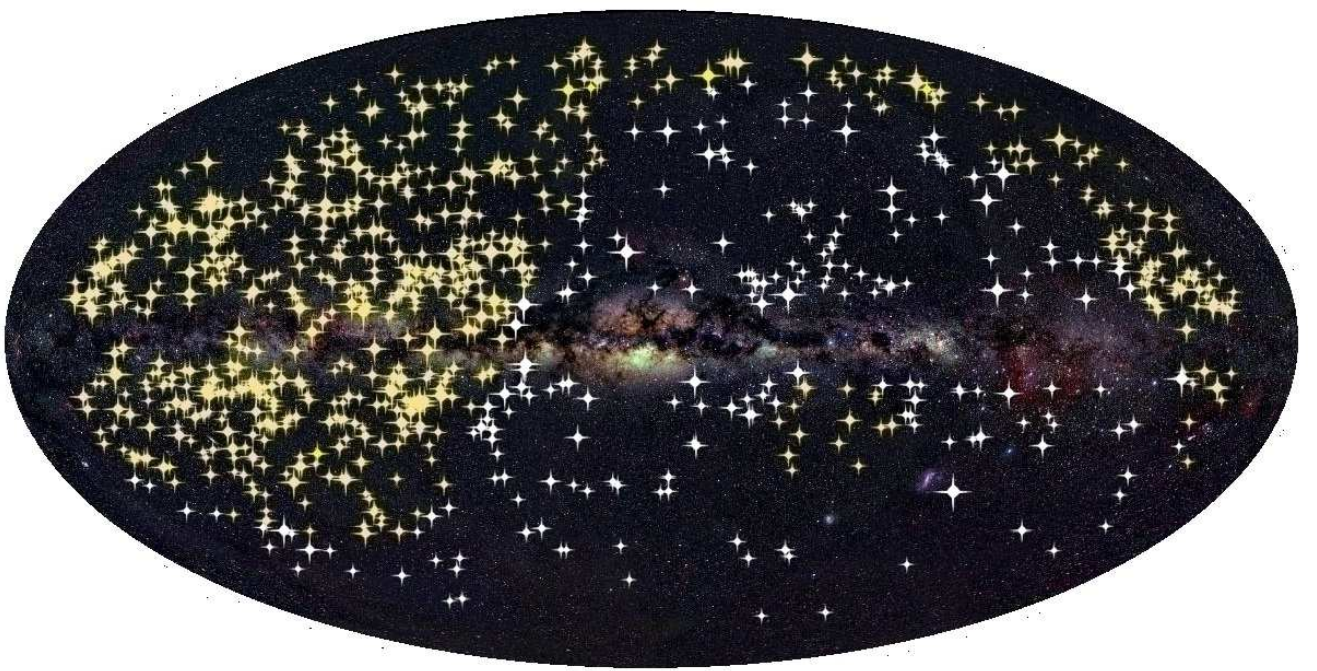

Figure 6: the distribution, in Galactic coordinate projection, of all the 886 MASTER transients discovered since the survey began in earnest in 2010. The white points represent the 141 transients discovered since MASTER-SAAO has been operational (Dec 2014 - Sep 2015). Size of star symbols scale with the amplitude of the transient.

\section{Cataclysmic Variable discoveries from MASTER}

In Figures $7 \& 8$ we compare the distributions (in Galactic coordinates) of the $855 \mathrm{CVs}$ discovered by CRTS [11] during the period 8 Nov 2007 to 31 July 2012 (4.7 years) and the 111 CVs discovered by MASTER-SAAO in the period 16 Dec 2014 to 5 Sep 2015 (0.7 years). Given the CRTS survey represents a $6.5 \times$ longer time span compared to MASTER-SAAO survey, the detection efficiencies are quite comparable, particularly given MASTER-SAAO 
represents just one site, while the CRTS CV survey includes results from 2 northern hemisphere sites and one southern hemisphere site (SSS). The total number of CVs discovered by all MASTER nodes totals 530, over a period of $\sim 5.75$ years. The contribution of MASTER-SAAO to this total is $\sim 20 \%$, although the time span for the latter is only $13 \%$ of total time elapsed since MASTER network began operating. These figures demonstrate how much more efficient the MASTER-SAAO node is in detecting transients, including CVs, compared to the other MASTER nodes, no doubt due to the lower sensitivity limit and overall better observing conditions. This was demonstrated when the detection limit achieved for an observation of a GRB, where 20 co-added 180s exposures reached a magnitude limit of $22^{\mathrm{m}} .3$, was more than a magnitude fainter than previously achieved at other MASTER sites.

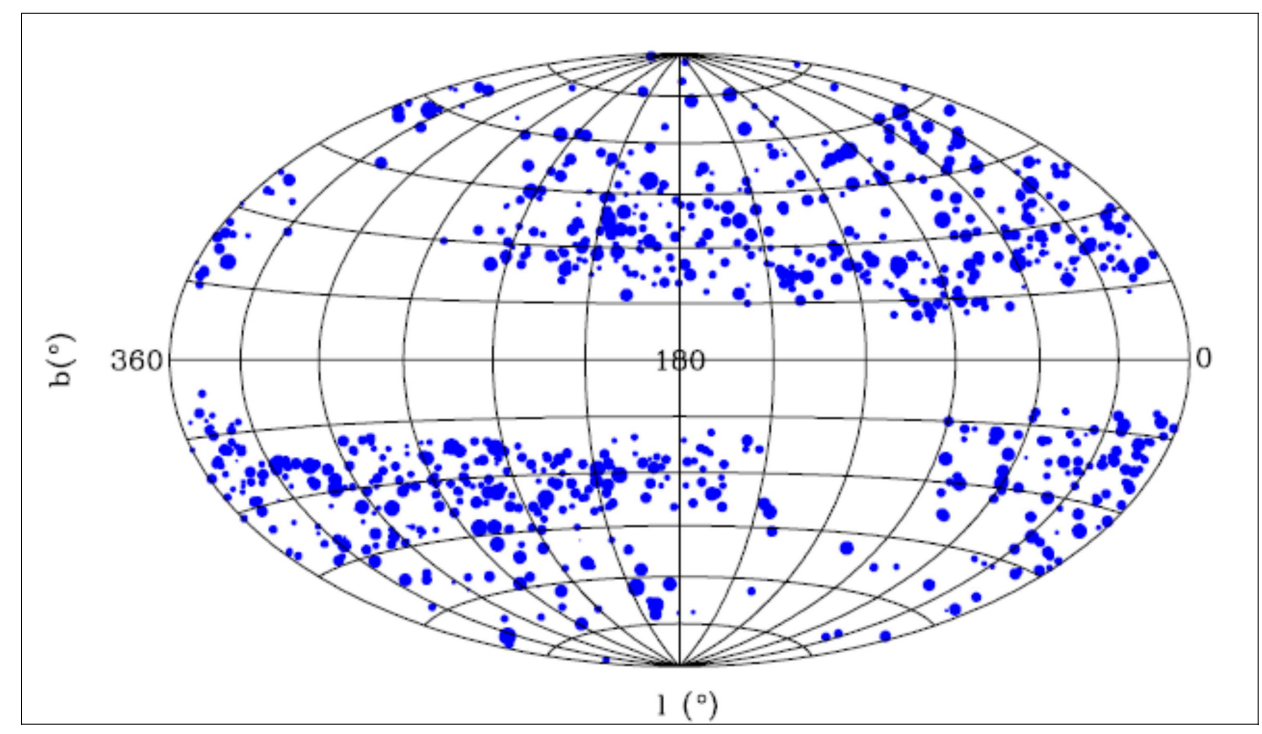

Figure 7: the distribution of all $855 \mathrm{CVs}$ discovered over 4.73 years by the CRTS [11], in Galactic coordinates, where the size of the blue circles scale with brightness.

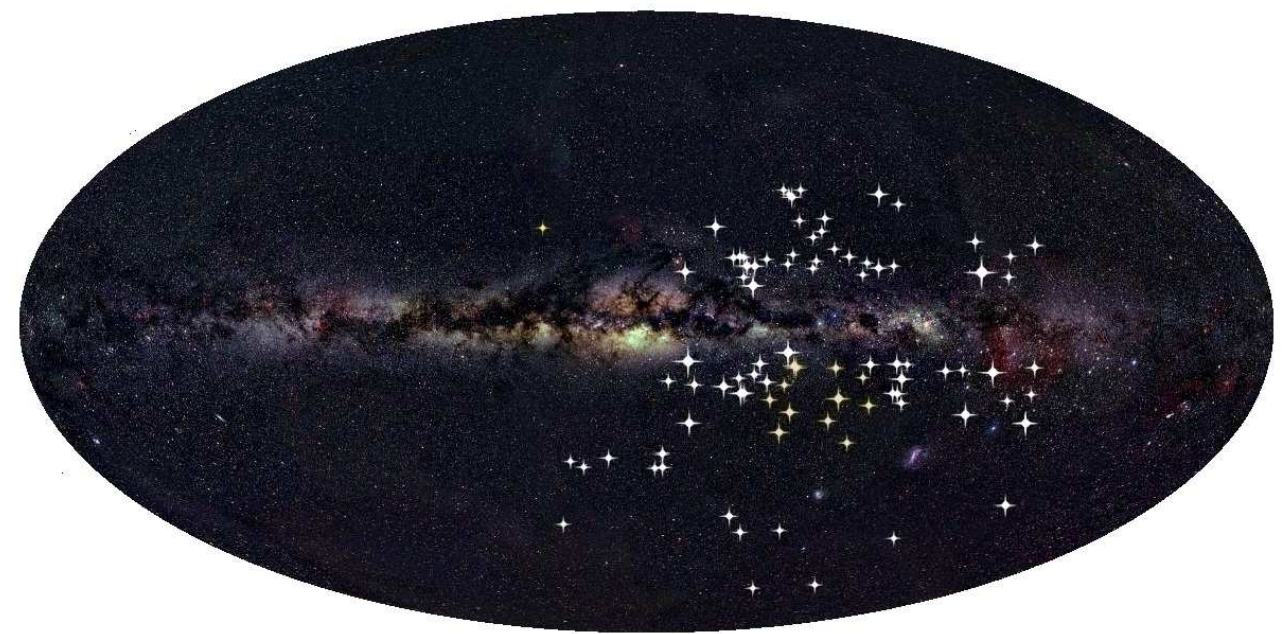

Figure 8: the distribution, in Galactic projection, of the $111 \mathrm{CVs}$ discovered in the $\sim 9$ months since MASTER-SAAO began operations. The size of symbols scale with outburst amplitude. 
In the study of the $855 \mathrm{CVs}$ in the CRTS [11], it was shown in figure 3 of that paper that the brightness histogram of the CVs discovered by CRTS peaked at a magnitude of $\mathrm{V}_{\mathrm{CSS}} \sim 17^{\mathrm{m}}$ and that there were essentially none with $\mathrm{V}_{\mathrm{CSS}}>20^{\mathrm{m}}$ (see Fig. 9). That study also had the benefit of having 150 previously known CVs in the sample and about half of the sample present in SDSS Data Release 8, thus providing additional colour information and spectra for some of the brighter candidates. The steep drop-off in the CV brightness histogram to fainter magnitudes contrasts to other classes of transients, e.g. Supernovae (SNe), where brighter (and closer) discoveries are relatively rare and they are typically much fainter (peaking at $\mathrm{V}_{\mathrm{CSS}} \sim 19$ ). The lack of equally faint CVs likely reflects the incompleteness of detection of the faintest DNe, with lowest amplitudes and/or longest outburst recurrence times.

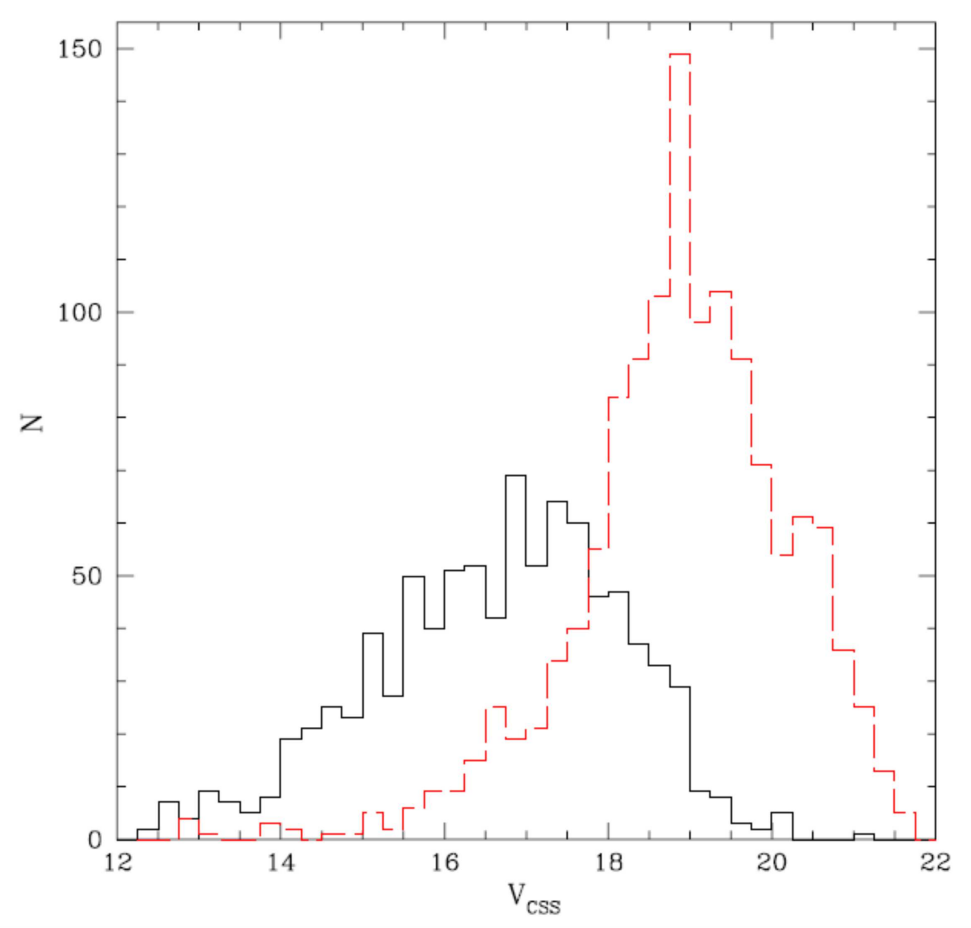

Figure 9: Histogram of the detection magnitudes of CRTS CVs (black) and SNe (red dashed), from [11]. The MASTER-SAAO limit is in the range $\mathrm{V}_{\mathrm{CSS}} \sim 19-20$, implying the two surveys are probably detecting the same population of CVs.

With a detection threshold of B 19-20, MASTER-SAAO, would seem to be sampling a similar population of CVs as CRTS has done to date [11]. Both surveys would appear to have similar selection effects in terms of sensitivity to outburst frequency and amplitude, which are determined by parameters like the survey cadence, image quality, resolution and the effectiveness of the automatic transient detection algorithm. The imposed amplitude threshold of $\sim 2.2^{\mathrm{m}}$ for MASTER-SAAO could well be impacting on the ability to detect fainter and lower amplitude DNe. However, while this may skew the statistics, the positive results from the survey is the detection of many new southern CVs/DNe, many with apparent large outburst amplitudes, as shown in Table 5. The MASTER-SAAO outburst amplitude results are quite similar to those of the CRTS [11], where the histogram of outburst amplitudes is shown in Fig. 
10 for the CRTS sample, based on both CRTS photometry and a smaller sample of 196 fainter CRTS CVs for which SDSS photometry exists, with consequently fainter limits. Based on a recent analysis of the statistical properties of CRTS DNe [13], those with amplitudes $>5^{\mathrm{m}}$ are all below the period gap (see figure 11 of [13]). This is an interesting population to study, since it includes the very short period and low accretion rate systems, with likely long outburst recurrence times, many near the period minimum, where there is an expected "pile up" of socalled period bouncers who have reached, or nearly reached the minimum period for CVs with non-degenerate secondaries. Of course there could also be some double-degenerate CVs (i.e. AM CVn systems) lurking amongst this faint short period population.

Table 5: MASTER-SAAO DNe outburst amplitudes

\begin{tabular}{|c|c|}
\hline Outburst magnitude & Percentage of DNe \\
\hline $2^{\mathrm{m}}-3^{\mathrm{m}}$ & $30 \%$ \\
\hline $3^{\mathrm{m}}-4^{\mathrm{m}}$ & $20 \%$ \\
\hline $4^{\mathrm{m}}-5^{\mathrm{m}}$ & $30 \%$ \\
\hline$>5^{\mathrm{m}}$ & $20 \%$ \\
\hline
\end{tabular}

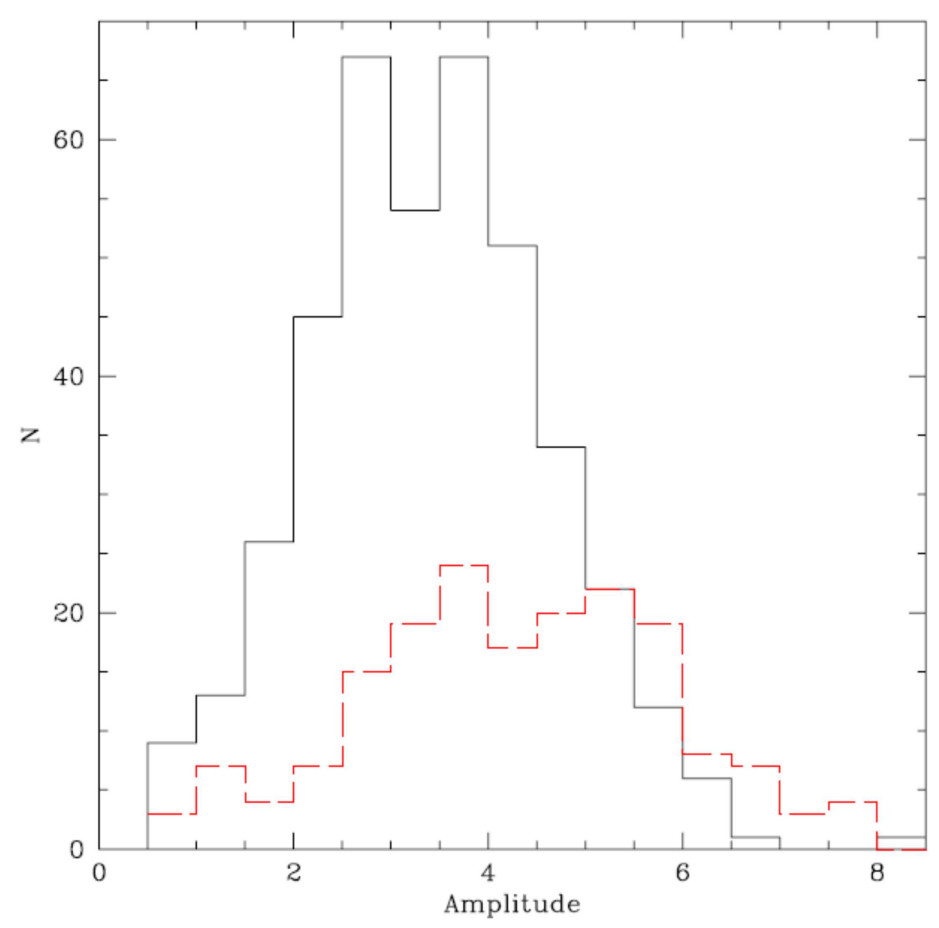

Figure 10: Histograms of the outburst amplitudes for CRTS DNe, where the solid line is the distribution for sources where the quiescent magnitude was bright enough to measure in Catalina data. The dashed line shows the distribution for fainter sources, where their SDSS $i$-band magnitude is assumed to be equivalent to the $V_{\mathrm{CSS}}$ quiescent brightness (from [11]). 
In the following two subsections we discuss some follow-up observations of two new CVs discovered by MASTER-SAAO. This provides a "flavour" of the type of follow-up observations of CVs which will be conducted in future with SALT and other SAAO facilitities.

\subsection{Dwarf Novae}

The majority of all the new MASTER-SAAO transient detections ( $78 \%)$ have been new Cataclysmic Variables, mostly Dwarf Novae (DNe), which undergo recurrent optical outbursts due to accretion disc instability cycles. While the threshold for an alert by MASTER is $\sim 2^{\mathrm{m}}$, the amplitudes for many of these new DNe (20\%) are well in excess of $5^{\mathrm{m}}$. The higher amplitude DNe are likely to be lower luminosity (so fainter), short orbital period $(<2 \mathrm{~h})$ systems of the SU UMa sub-group, with the WZ Sge stars being the faintest, highest amplitude, lowest accretion rate and shortest period systems of all of them. These characteristics, coupled with their infrequent outbursts (up to decades), make them difficult to detect and study. However, they may well represent a "hidden iceberg" population of CVs, which are important in understanding $\mathrm{CV}$ evolution, particularly at the extreme short orbital period limit, where gravitational radiation drives the angular momentum loss in these systems. So finding new examples is an important result.

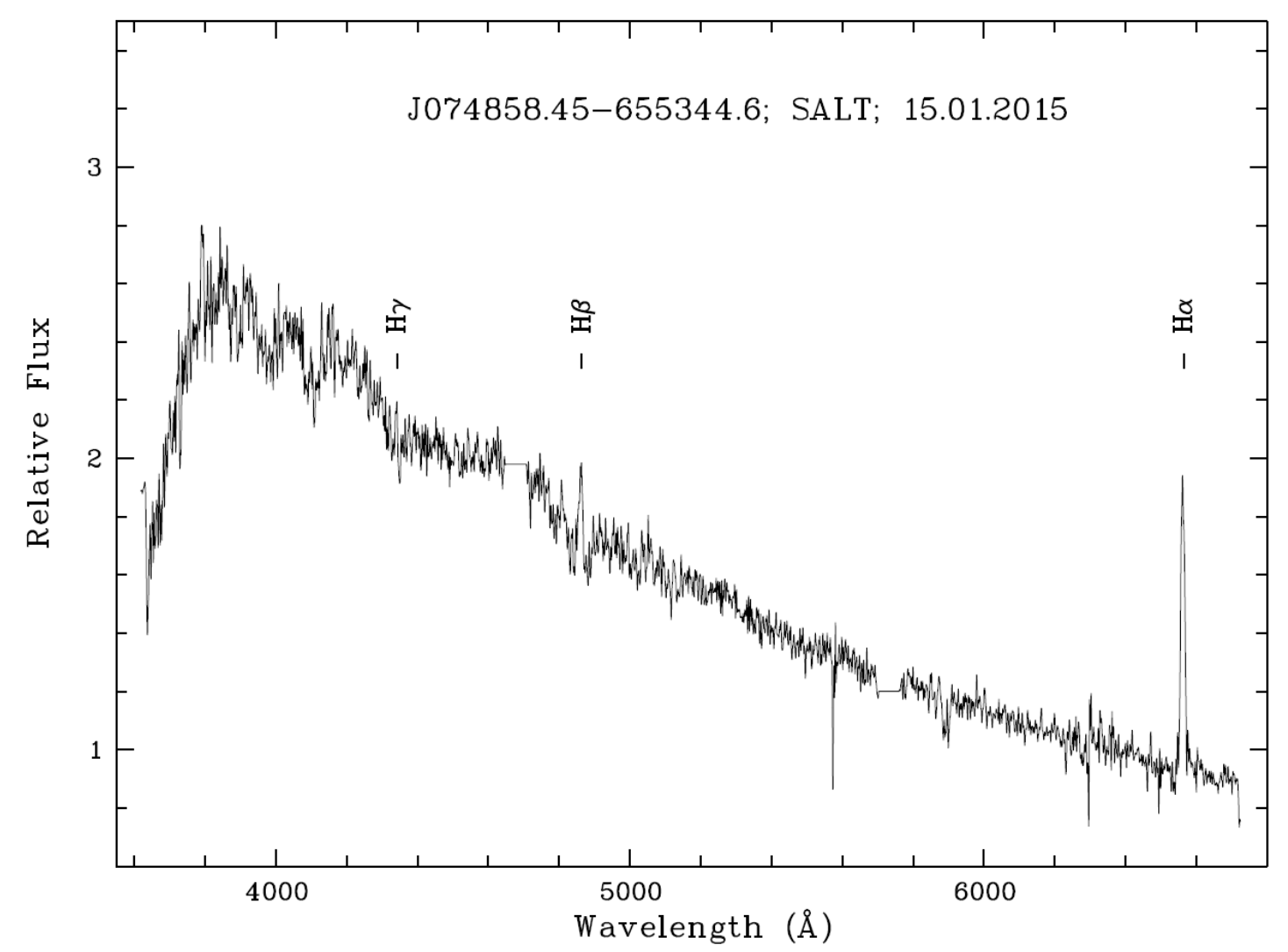

Figure 11: SALT RSS spectrum of the new Dwarf Nova MASTER_OT_J074858.45-655344.6. 
One example of a newly identified DNe is MASTER_J074858.45-655344.6, which was discovered by MASTER-SAAO on 15 Jan 2015 as a $B \sim 17^{\mathrm{m}}$ magnitude transient with a $\sim 3^{\mathrm{m}}$ amplitude [14] during a search for the optical counterpart of GRB150114, discovered by Fermi. The optical transient was observed the following night with the SALT Robert Stobie Spectrograph (RSS) and a 1200s exposure spectrum, covering 3600-6600 $\AA$ at a resolution of 5.6̊̊, was obtained (see Fig. 11). The spectrum shows a rising blue continuum and strong $\mathrm{H} \alpha$ emission, with an E.W. $=16.6 \pm 0.4 \AA$ and FWHM $=12.2 \pm 0.3 \AA$. The higher order Balmer series show broad absorption lines with narrow emission cores, with the $\mathrm{H} \beta$ peak rising above the local continuum level, which is typical of a DNe declining from outburst, and leading to this classification [14]. Clearly this DNe transient was unrelated to GRB150114.

\subsection{The eclipsing polar MASTER OT J061451.70-272535.5}

This transient was discovered on 19 Feb 2015 at an unfiltered magnitude of $18^{\mathrm{m}} .3$ [15] and was observed photometrically at the SAAO using the $1.9 \mathrm{~m}$ and $1.0 \mathrm{~m}$ telescopes using the SHOC high speed EM-CCD cameras. Time resolved $B, V$ and unfiltered photometry, with $10-$ 45 s time resolution, was obtained over 5 nights [15]. Light curves revealed that the system eclipses, with a period of 2.08 hours. In addition, there are two minima, with an initial sharp drop, where the flux decreases by $\sim 50 \%$, before slowly increasing in brightness again. A second rapid ingress follows, where the system becomes totally eclipsed, followed by a sharp egress (see Fig. 12). This was interpreted [16] as evidence for initial obscuration of a bright accretion spot(s) on the white dwarf by the accretion stream, followed by the eclipse of the white dwarf and accretion spot(s) by the secondary star. The pre-eclipse dip is reminiscent of that observed in HU Aqr [17]. In addition there is some evidence the system has two accreting poles, due to a possible step in the egress.

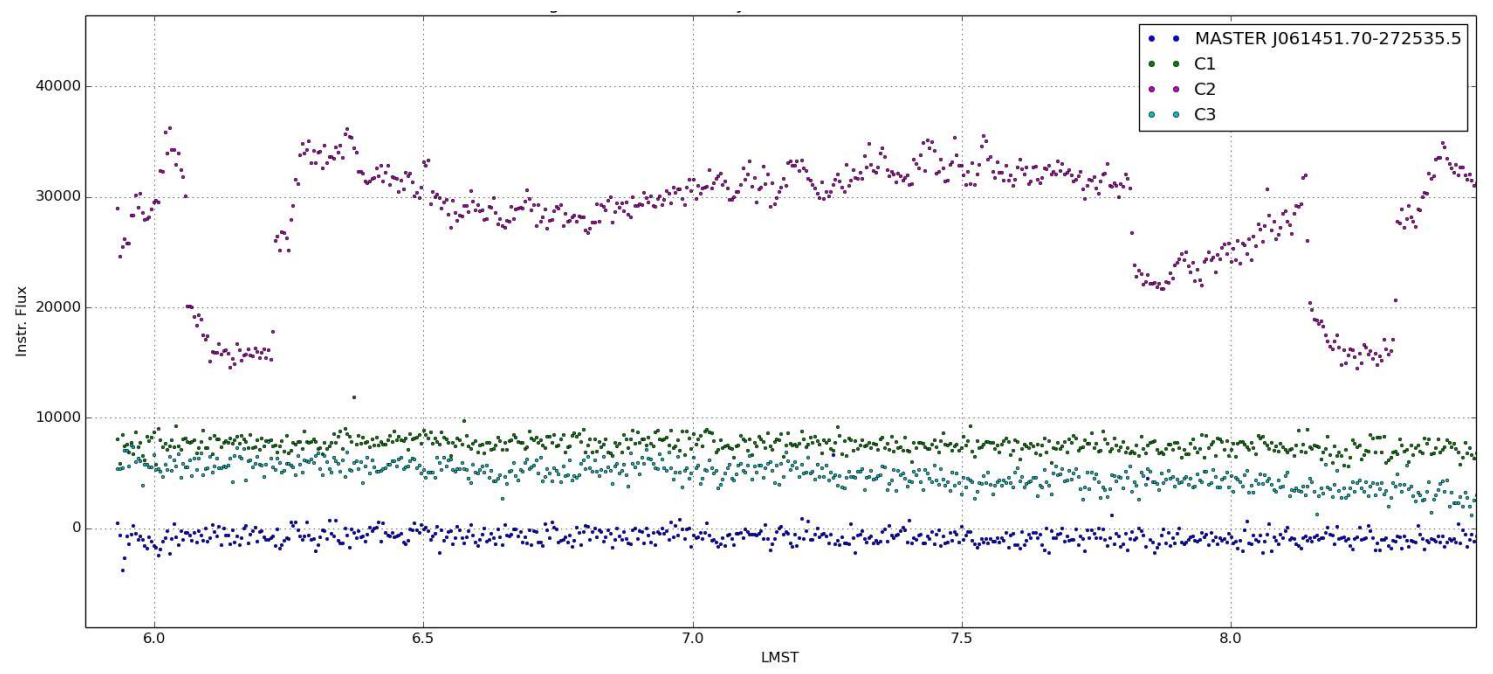

Figure 12: light curve (no filter) of MASTER_J061451.70-272535.5 taken on 24 Feb 2015 using the SHOC high speed photometer on the SAAO 1.9-m telescope. The eclipse and pre-eclipse dip, due to the obscuring accretion stream, is clearly seen. The points at the bottom of the plot are the fluxes of three comparison stars, which have been offset for clarity. 
The target was subsequently observed with SALT RSS on 28 Feb 2015 and a $800 \mathrm{~s}$ exposure spectrum obtained, covering 4060-7120 (see Fig.13). The spectrum shows all of the Balmer lines in emission, a strong HeII $4686 \AA$ line at a peak flux greater than $\mathrm{H} \beta$, and weaker HeI lines. The lines appear asymmetric and double peaked, with wings extending to shorter wavelengths. This spectrum is typical of a magnetic Cataclysmic Variable, particularly with the strong HeII line, and therefore together with the light curves, we have identified the system to be a new eclipsing polar [16].

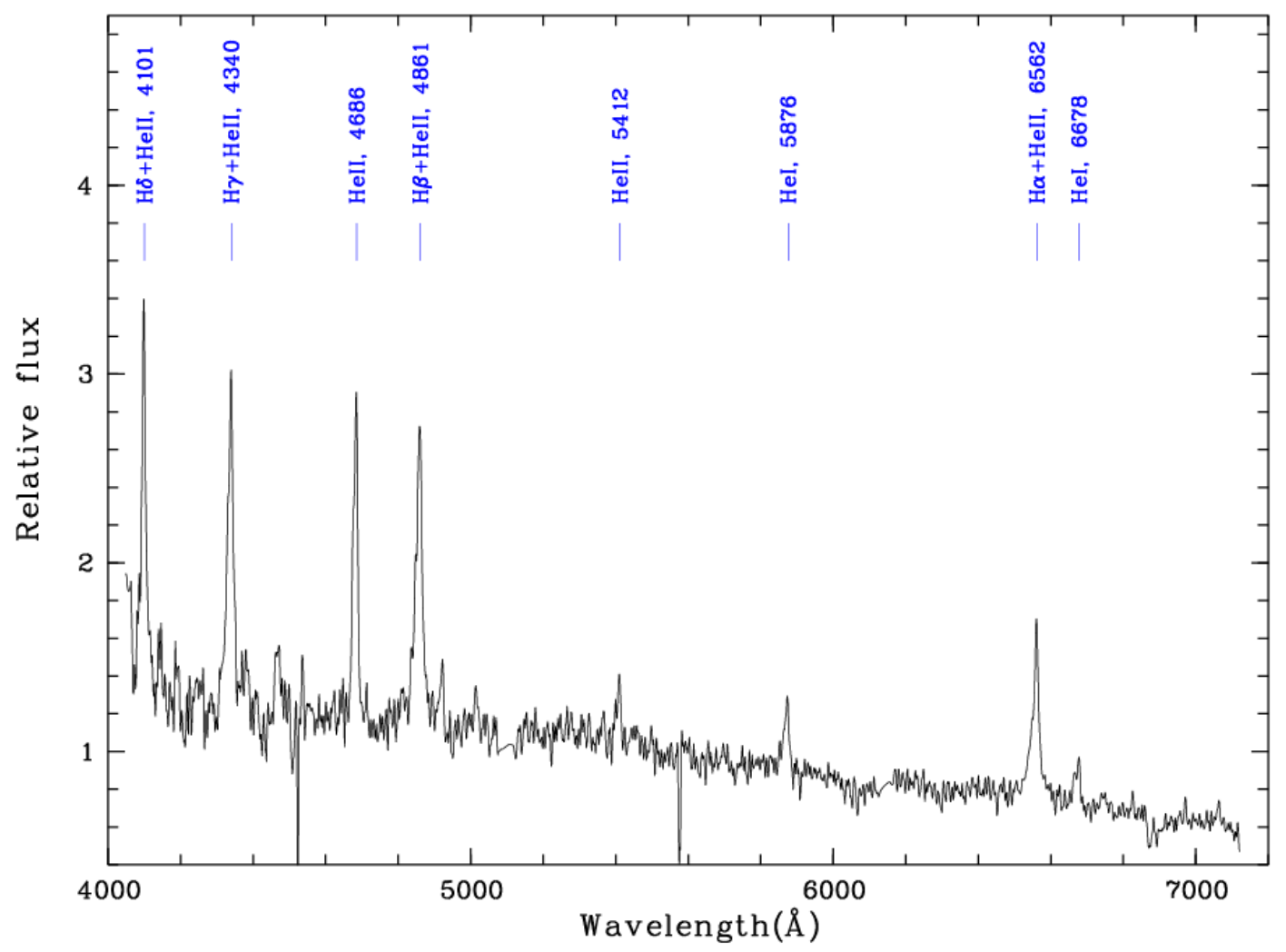

Figure 13: A SALT RSS spectrum of the eclipsing polar, MASTER OT J061451.70-272535.5. Note the prominent high excitation emission line of HeII $\lambda 4686 \AA$, typical of magnetic CVs.

\section{Future Strategies for follow-up of transients with SAAO facilities}

While MASTER-SAAO is the first local optical transient detection system to be installed at the Sutherland station of the SAAO, it will be joined in 2016 by another transient detection facility, namely MeerLICHT (http://www.ast.uct.ac.za/meerlicht/MeerLICHT.html). This will be a $0.65 \mathrm{~m}$ telescope with a 2 degree $^{2}$ field of view, which will simultaneously observe the same fields being observed by the MeerKAT radio telescope, once it begins operations. The aim is to have simultaneous optical images of MeerKAT fields to allow for the potential detection of the optical counterparts of radio transients. 
Many transient discoveries, including CVs, require follow-up observations to confirm their nature and to allow for more probing astrophysical observations. While there have already been several examples of limited follow-up observations (e.g. by SALT and other SAAO telescopes) presented in the previous section, further plans are underway to expand the nature of these follow-up observations. These include more comprehensive observations of the whole variety of optical transients currently being detected by the MASTER and other networks, including CVs, GRBs, AGN and X-ray and $\gamma$-ray transients. A new SALT program supporting this began in Nov 2015, while observations with other multi-wavelength facilities are also planned, including HESS II, ASTROSAT (the new Indian optical/UV/X-ray satellite) and eventually MeerKAT, once it begins operating (2016/17). In addition, new optical follow-up possibilities will be enabled with the establishment of two new robotic facilities at the SAAO, namely MONETSouth and the new $1.0 \mathrm{~m}$ Robotic telescope.

MONET-South, owned by the University of Göttingen in Germany (see https://monet.unigoettingen.de/), is the twin telescope to MONET-North, situated at MacDonald Observatory in Texas, USA. Both are robotic/remotely operated $1.2 \mathrm{~m}$ telescopes, with a major science goal being the follow-up of transients and ToOs. MONET-North has been operating for several years and MONET-South at Sutherland is expected to begin science operations shortly, initially with an imaging camera. A project has currently begun to design and construct an efficient fibre fed low resolution spectrograph (MORISOT). With such an instrument, MONET-South will have the capability to rapidly spectroscopically classify many brighter $(V<\sim 16-17)$ optical transients as well as supporting coordinated multi-wavelength ToO observations.

The new SAAO $1.0 \mathrm{~m}$ Robotic telescope, which is currently under construction and is being provided by APM in Germany, is expected to be installed at Sutherland in 2016. While the final instrument suite is still to be determined, it will at least initially consist of an imaging camera and high speed EM-CCD camera and, eventually it is hoped, fibre fed low and or high resolution spectrographs. Science goals for this telescope include synoptic monitoring of variable objects (stars and AGN) at a variety of cadences, but also supporting follow-up observations of transients and ToO.

All of the follow-up facilities at SAAO will require automated response to transient and ToO alerts that avoids the slow response typical of human initiated observations. Fortunately there exist several robotic telescope observing software protocols [18] (e.g. the RTML protocol [19]) which can efficiently trigger robotic telescopes to conduct follow-up observations once an alert is received (e.g. from the likes of VO Events [18]). A project to implement the automated follow-up observations from transient alerts has begun, which should be implemented during 2016.

\section{Summary and Final Remarks}

In this paper we have presented a review of the new optical transient detection system at the Sutherland observing station of the SAAO, namely MASTER-SAAO, the first MASTER II system established in the southern hemisphere, which began operations in mid-Dec 2014. We have reviewed the specifications and modus operandi for the MASTER system, and particularly MASTER-SAAO, and its survey for transients, focusing on the results of new CV discoveries. 
In the $\sim 9$ months of operations, MASTER-SAAO has discovered 141 new non-Solar System optical transients (in addition to two comets and several minor planets) during its survey mode. In addition it has conducted follow-up observations of $\sim 10$ other transient detections, originating from other alert systems (e.g. Swift, Fermi), including GRB afterglow searches and monitoring of AGN [20].

A total of 111 new CVs have been discovered with MASTER-SAAO, a significant advance and resulting in a total of 530 new CVs discovered by the entire MASTER network in its 5.7 years of operations. The study conducted on the CVs detected in the CRTS [11] demonstrated that most new CVs discovered through transient detection systems are Dwarf Novae, with average outburst amplitudes of $\sim 4^{\mathrm{m}}$, which is very similar to the statistics of the MASTER-SAAO results to date. We conclude that the two CV surveys are very similar, with the two samples pushing the limiting magnitude deeper and consequently revealing a somewhat different CV population compared to the brighter systems discovered previously (e.g. from spectroscopic surveys). The mean magnitude of the new CVs found by CRTS is $V_{\text {CSS }} \sim 17$, with very few system fainter than $V_{\text {CSS }} \sim 20$. This is likely due to selection effects, where fainter and lower amplitude DNe are more difficult to detect. With MASTER having a similar limiting magnitude to CRTS ( $V_{\text {CSS }} \sim 20$ ), we believe that the two CV populations discovered by these complementary surveys are therefore quite similar. Now that the SSS (Australia) node of the CRTS has ceased operating, only MASTER-SAAO is continuing to systematically survey the southern skies for new CVs, although more CV discoveries are also following from surveys for other types of transients, for example SNe from the likes of the ASAS-SN survey.

The results of two follow-up observations on two CVs were reported, including spectroscopic observations with SALT using Director's Discretionary Time. More detailed papers describing these results and their interpretation are currently in preparation and more intensive SALT follow-up observations of transients has begun, including of new CV discoveries.

Finally we discussed the future possibilities of more expansive programs on transient follow-up observations at SAAO, exploiting two new robotic facilities that will become operational during 2016. Our experiences gained in these endeavours will help to inform how to conduct larger scale SALT and SAAO transient follow-up campaigns in the future, for example with the expansive transient programs associated from the SkyMapper, GAIA and LSST missions.

\section{Acknowledgements}

We thank the SAAO Director, Profesor T. Williams, for supporting the establishment of the MASTER-SAAO node at the SAAO. We are also grateful for the support of MASTER-SAAO from the SAAO technical staff, in particular J. Stoffels, H. Kamfer, C. Fourie and P. Fourie. This program is partially supported through the SA-GAMMA group and by the National Research Foundation of South Africa, through research support grants to DB, HB, MM, AK, MMK and SBP. Some of the observations presented here were carried out on the Southern African Large telescope (SALT) through an allocation of Directors Discretionary Time, for which we are most grateful. 


\section{References}

[1] N. Law, et al., 2009, The Palomar Transient Factory: System Overview, Performance, and First Results, PASP, 121, 1395

[2] B. J. Shappee, et al., 2014, The Man behind the Curtain: X-Rays Drive the UV through NIR Variability in the 2013 Active Galactic Nucleus Outburst in NGC 2617, ApJ, 788, 48

[3] C. W. Akerlof, et al., 2003, The ROTSE-III Robotic Telescope System, PASP, 115, 132

[4] G. Melady, et al., 2009, Watcher Robotic Telescope Follow-Ups of GRBs, AIP Conf. Proc. 1133, 67

[5] A. J. Drake, et al., 2009, First Results from the Catalina Real-Time Transient Survey, ApJ, 696, 870

[6] V. Lipunov, et al., 2010, MASTER Robotic Net, Advances in Astronomy, Volume 2010, Article ID 349171

[7] K. W. Hodapp, et al., 2004, Optical design of the Pan-STARRS telescopes, Proc. SPIE, 5489, 667

[8] S. Keller, et al., 2007, The SkyMapper Southern Sky Survey, PASA, 24, 1

[9] M. A. C. Perryman et al., 2001, GAIA: Composition, formation and evolution of the Galaxy, A\&A, 369,339

[10] Z. Ivezic et al., 2008, LSST: from Science Drivers to Reference Design and Anticipated Data Products, preprint (astro-ph/0805.2366)

[11] A.J. Drake, et al., 2014, Cataclysmic variables from the Catalina Real-time Transient Survey, MNRAS, 441, 1186.

[12] V. G. Kornilov et al, 2012, Robotic optical telescopes global network MASTER II. Equipment, structure, algorithms, Exp Astron 33:173-196

[13] D. Coppejans, et al., 2015, Statistical properties of dwarf novae-type cataclysmic variables: The outburst catalogue, MNRAS, in press [arXiv:1215.03821]

[14] D.A.H. Buckley, et al., 2015, SALT spectral classification of MASTER OT J074858.45-655344.6.5 as a likely Dwarf Nova in outburst, ATel \#7165

[15] V. Shumkov, et al., 2015, MASTER: 4 optical transients discovery, ATel \#7127

[16] D.A.H. Buckley, et al., 2015, Classification of MASTER OT J061451.70-272535.5 as an eclipsing Polar, ATel \#7169

[17] M.K. Harrop-Allin, et al., 1999, Indirect imaging of the accretion stream in eclipsing polars -II. HU Aquarii, MNRAS, 308, 807.

[18] A. Klotz, 2010, Protocols for Robotic Telescope Networks, Advances in Astronomy, Volume 2010, Article ID 496765, 8 pages

[19] F. V. Hessman, 2006, Remote telescope markup language (RTML), Astronomische Nachrichten, vol. 327, no. 8, pp. 751-757.

[20] D.A.H. Buckley, et al., 2016, Detecting and Observing Transients \& Targets of Opportunity at $S A A O \& S A L T$, Proceedings of HEASA-2015 conference, Proceedings of Science, in press. 